\title{
Physiological functions of Atg6/Beclin 1: a unique autoph- agy-related protein
}

\author{
Yang Cao ${ }^{1}$, Daniel J Klionsky ${ }^{1}$ \\ ${ }^{I}$ Departments of Molecular, Cellular and Developmental Biology and of Biological Chemistry, Life Sciences Institute, University of \\ Michigan, Ann Arbor, Michigan 48109-2216, USA
}

The most striking morphological feature of eukaryotic cells is the presence of various membrane-enclosed compartments. These compartments, including organelles and transient transport intermediates, are not static. Rather, dynamic exchange of proteins and membrane is needed to maintain cellular homeostasis. One of the most dramatic examples of membrane mobilization is seen during the process of macroautophagy. Macroautophagy is the primary cellular pathway for degradation of long-lived proteins and organelles. In response to environmental cues, such as starvation or other types of stress, the cell produces a unique membrane structure, the phagophore. The phagophore sequesters cytoplasm as it forms a double-membrane cytosolic vesicle, an autophagosome. Upon completion, the autophagosome fuses with a lysosome or a vacuole in yeast, which delivers hydrolases that break down the inner autophagosome membrane along with its cargo, and the resulting macromolecules are released back into the cytosol for reuse. Autophagy is therefore a recycling process, allowing cells to survive periods of nutrient limitation; however, it has a wider physiological role, participating in development and aging, and also in protection against pathogen invasion, cancer and certain neurodegenerative diseases. In many cases, the role of autophagy is identified through studies of an autophagy-related protein, Atg6/Beclin 1. This protein is part of a lipid kinase complex, and recent studies suggest that it plays a central role in coordinating the cytoprotective function of autophagy and in opposing the cellular death process of apoptosis. Here, we summarize our current knowledge of Atg6/Beclin 1 in different model organisms and its unique function in the cell.

Keywords: lysosome, phosphoinositides, protein targeting, stress, vacuole, yeast

Cell Research (2007) 17:839-849. doi: 10.1038/cr.2007.78; published online 25 September 2007

\section{Introduction}

There are various processes that fall under the general name of autophagy, which has the general definition of

Correspondence: Daniel J Klionsky

Tel: +1-734-615-6556; Fax: +1-734-763-6492

E-mail: klionsky@umich.edu

Abbreviations: activating molecule in Beclin 1-regulated autophagy (Ambra1); autophagy-related (Atg); Bcl-2-homology-3 (BH3); coiled-coil domain (CCD); cytoplasm to vacuole targeting (Cvt); evolutionarily conserved domain (ECD); embryoid bodies (EB); endoplasmic reticulum (ER); hypersensitive response (HR); herpes simplex virus type 1 (HSV-1); Niemann-Pick $\mathrm{C}$ (NPC); programmed cell death (PCD); phosphatidylethanolamine (PE); phosphatidylinositol (PtdIns); UV irradiation resistance-associated gene (UVRAG); vacuolar protein sorting (Vps) any process involving the degradative delivery of a portion of the cytoplasm to the lysosome (or its yeast analog, the vacuole) that does not involve direct transport through the endocytic or vacuolar protein sorting (Vps) pathways [1]. The best characterized of these processes is macroautophagy, which we refer to hereafter as autophagy. Autophagy is highly conserved in eukaryotic cells, ranging from yeast to mammals [2]. It is the major intracellular pathway for degradation and recycling of long-lived proteins and organelles, whereas the ubiquitin/proteasome system degrades primarily short-lived proteins.

One of the most distinctive features of autophagy is its relatively unlimited capacity for degradation, which reflects the unique mechanism of cargo sequestration. During autophagy, a membrane of unknown origin forms 
a nucleation site that generates a unique organelle, the phagophore (Figure 1). The phagophore expands, probably through vesicular addition, to form a large double-membrane cytosolic vesicle that is termed an autophagosome. Unlike transient transport vesicles such as those that shuttle proteins between the organelles of the secretory pathway, the autophagosome forms de novo; it does not bud off intact from a pre-existing organelle, although the membrane that adds to the growing phagophore likely includes vesicles that originate within the secretory pathway. This expansion mechanism allows the autophagosome to sequester entire organelles or large protein aggregates, and even invading pathogens. Following completion of the autophagosome, it fuses with the lysosome or the yeast vacuole. In mammalian cells, autophagy also converges with endocytosis, a form of heterophagy, and the autophagosome may fuse with an endosome to generate an amphisome, which also will ultimately fuse with a lysosome; it is not known whether

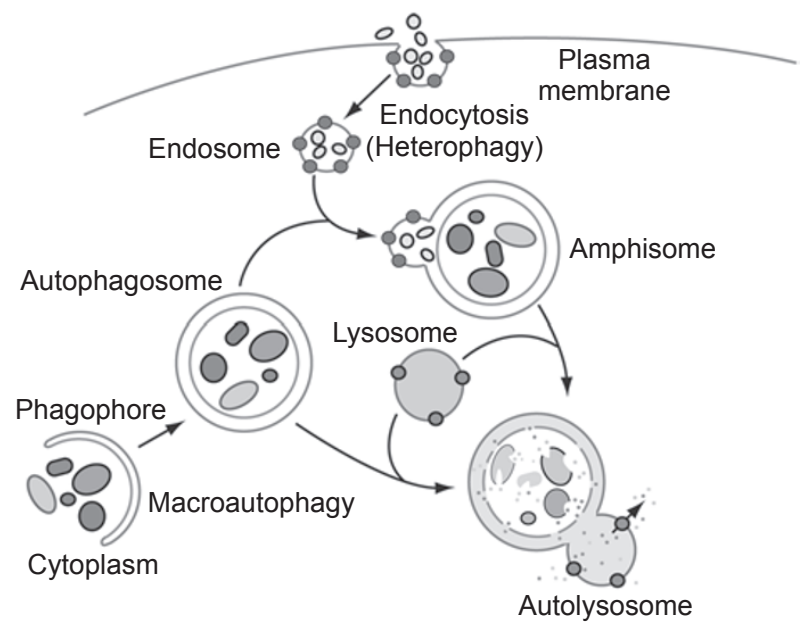

Figure 1 A schematic model of macroautophagy in mammalian cells. Autophagy occurs at a low, constitutive basal level, but can be induced by environmental signals including stress (e.g., nutrient depletion) and hormones (e.g., glucagon). The process begins with the formation of a sequestering membrane termed a phagophore. During nonspecific autophagy, bulk cytoplasm (including entire organelles) can be sequestered, whereas specific types of autophagy can target selective cargos. The phagophore expands to form a doublemembrane autophagosome. The autophagosome may fuse with an endosome; convergence of endocytosis, a form of heterophagy, and autophagy in the form of a fusion event generates an amphisome. The amphisome or autophagosome acquires hydrolytic enzymes by fusing with a lysosome. The resulting autolysosome breaks down the inner membrane of the autophagosome and its cargo. The macromolecular breakdown products are released back into the cytosol through permeases for reuse. this process occurs in yeast because the endosome is poorly defined and difficult to detect. In either case, the final fusion event provides access to the diverse hydrolases that reside in the lysosome and yeast vacuole, allowing them to break down the inner membrane of the autophagosome along with its cargo. The resulting macromolecules are then released back into the cytosol for reuse.

Why does the cell maintain a process for eating parts of itself? For proper physiology, the cell must maintain a balance between anabolism and catabolism. Autophagy is a catabolic process that has homeostatic functions, acting in the turnover of various worn out or damaged macromolecules and organelles [3]. Autophagy also has cytoprotective roles during stress conditions, such as nutrient starvation, growth factor depletion [4] and pathogen invasion [5] that might otherwise lead to apoptosis or type I programmed cell death (PCD). For example, damaged mitochondria pose a threat to cell stability due to the generation of excess reactive oxygen species that can cause nuclear DNA damage [6, 7]. The compromised organelle may release cytochrome $c$ into the cytosol, which can trigger apoptosis as a barrier against oncogenesis. If the cell can remove the damaged organelle in a timely manner, however, it may avoid the need for apoptosis. Thus, although we are not aware of evidence demonstrating a direct connection between the removal of damaged organelles and the prevention of apoptosis, it seems likely that autophagy can protect the cell against apoptotic death through its action as an organelle qualitycontrol mechanism.

On the other hand, autophagy presents us with a challenging conundrum because it sometimes acts as a killer of cells by promoting autophagic, or type II, PCD with the hallmark of accumulated autophagosomes in dying cells [8]. In this case, extensive autophagy may participate along with, or instead of, apoptosis to ensure the death and removal of cells that cannot be rescued; it is not difficult to imagine that an excessive level of self-digestion would be deleterious. The dual role of autophagy, in cytoprotection and cell death, is one of the most fascinating features of this process, and one that needs to be better understood if we ever hope to harness autophagy for therapeutic use. Indeed, there are many possibilities for modulating autophagy for purposes of human health: autophagy has been implicated in various human diseases, including cancer, cardiomyopathy and the prevention of certain neurodegenerative disorders [9-11].

In the last decade, with the identification of approximately 30 AuTophaGy-related (ATG) genes in Saccharomyces cerevisiae and other fungi [12], the molecular mechanisms of autophagy have gradually been elucidated [2]. As mentioned above, autophagy is conserved across all eukaryotes and homologs of many yeast $A T G$ genes have recently been 
identified in various eukaryotic systems, and the underlying molecular mechanisms of autophagy are also conserved. Among the $A T G$ genes, $A T G 6$ is relatively unique in its not being "autophagy-specific." For example, the $S$. cerevisiae $A T G 6 / V P S 30$ gene product is the only protein required for both autophagy and sorting of the vacuole resident hydrolase carboxypeptidase $\mathrm{Y}$ through the Vps pathway [13]. Of the Arabidopsis atg (atatg) mutant plants examined so far, only those with defects in AtATG6 are defective in pollen germination $[14,15]$. Beclin 1, the mammalian homolog of yeast $A T G 6$, is a haploinsufficient tumor suppressor gene $[16,17]$. Finally, the Beclin 1 protein is also an important convergence point of autophagy and apoptosis; it interacts with anti-apoptotic Bcl-2-like proteins [18], and has recently been found to be a Bcl-2-homology-3 (BH3)-only protein $[19,20]$.

In the past decade, a substantial amount of research has been carried out on the Atg proteins, and several recent reviews discuss the protein machinery of autophagy [21-24]. However, despite the growing interest in Atg6/Beclin 1 and its central role in autophagic regulation, no review is available on this specific topic. Here, we summarize what we know about Atg6/Beclin 1 from studies in S. cerevisiae, plants, Caenorhabditis elegans, mice and mammalian cell lines, with an emphasis on its uniqueness compared to other Atg proteins. Finally, the role of Atg6/Beclin 1 in autophagic and apoptotic cell death is discussed.

\section{Atg6/Vps30 participates in autophagy and the Vps pathway}

To date, we have been referring to macroautophagy as one process; however, just as there are different types of autophagy, there are also distinct types of macroautophagy. In general, macroautophagy is considered to be nonspecific, but there are also specific types of macroautophagy. For example, excess peroxisomes can be selectively degraded through a specific macroautophagy-like process termed pexophagy [25]. One well-characterized selective type of autophagy is the cytoplasm to vacuole targeting (Cvt) pathway that is used for the delivery of two vacuolar hydrolases, aminopeptidase I and $\alpha$-mannosidase [26]. The protein machinery of nonspecific autophagy overlaps extensively with the Cvt pathway [27, 28]; however, there are some proteins that are specific for each pathway. All of the proteins that are needed for both the Cvt pathway and autophagy appear to be specifically engaged in autophagyrelated pathways, except for Atg6/Vps30 and Atg18 [29]. A brief summary of the functions of the Atg proteins is presented in Table 1.

As noted above, aminopeptidase I and $\alpha$-mannosidase are delivered to the vacuole through the autophagy-related
Cvt pathway. In contrast, the majority of vacuolar hydrolases (including carboxypeptidase $\mathrm{Y}$ ) are delivered to their final destination primarily through the Vps pathway [30]. An obvious question that arises is why Atg6/Vps30 is involved in these two separate pathways. One type of complex that is involved in regulating autophagy is a lipid kinase, in particular a phosphatidylinositol (PtdIns) 3-kinase. In mammals, there are two different PtdIns 3-kinases that participate in autophagy regulation: the class I PtdIns 3kinase generates PtdIns $(3,4,5) \mathrm{P}_{3}$ and is inhibitory, whereas the class III enzyme generates PtdIns(3)P and is stimulatory [31]. Yeast $A \operatorname{tg} 6 / \mathrm{Vps} 30$ is a subunit of two distinct class III PtdIns 3-kinase complexes [32] (Figure 2). Complex I functions in autophagy, whereas complex II is involved in Vps, which explains why Atg6/Vps30 participates in both, otherwise separate, pathways.

The two PtdIns 3-kinase complexes contain three common subunits: the PtdIns 3-kinase enzyme Vps34, the regulatory protein Vps15 and Atg6/Vps30. In addition, each complex has a specific factor, Atg14 for complex I and Vps38 for complex II. Atg14 directs complex I to the phagophore assembly site, also termed the pre-autophagosomal structure (PAS) [33], a perivacuolar site where most of the Atg proteins colocalize at least transiently and autophagosomes are thought to originate [34, 35]. Moreover, Atg14 together with Atg6 are important in mediating the localization of other Atg proteins to the PAS [35, 36]. In contrast, Vps38 is responsible for the endosomal localization of complex II [33]. In atg14 $v$ ps $38 \Delta$ cells, Vps34 and Vps15 still localize to some punctate structures (neither endosomes nor the PAS) and vacuolar membranes, indicating that potential novel PtdIns 3-kinase complexes exist [33]. It is of interest to know what other components, if any, are included in these putative novel complexes and to determine their specific function. Candidate mammalian homologs of Atg14 and Vps38 have recently been identified ( $\mathrm{N}$ Mizushima and T Yoshimori, personal communication). Considering the conserved roles of class III PtdIns 3-kinases in autophagy and lysosomal or vacuolar protein sorting, it is not surprising that different Beclin 1-containing complexes also exist in mammalian cells.

\section{Beclin 1 is a mammalian tumor suppressor}

Bcl-2 is an anti-apoptotic protein [37], and Beclin 1 was first identified as a Bcl-2-interacting protein in a yeast twohybrid screen [18]. The beclin 1 gene is monoallelically deleted in up to $75 \%$ of ovarian, $50 \%$ of breast and $40 \%$ of prostate cancers [38]. Decreased expression of Beclin 1 is also observed in other types of cancers including human brain tumors [39] and cervical cell carcinoma [40]. Expression of Beclin 1 in human MCF7 breast carcinoma cells 
Table 1 Proteins required for specific and nonspecific autophagy in yeast

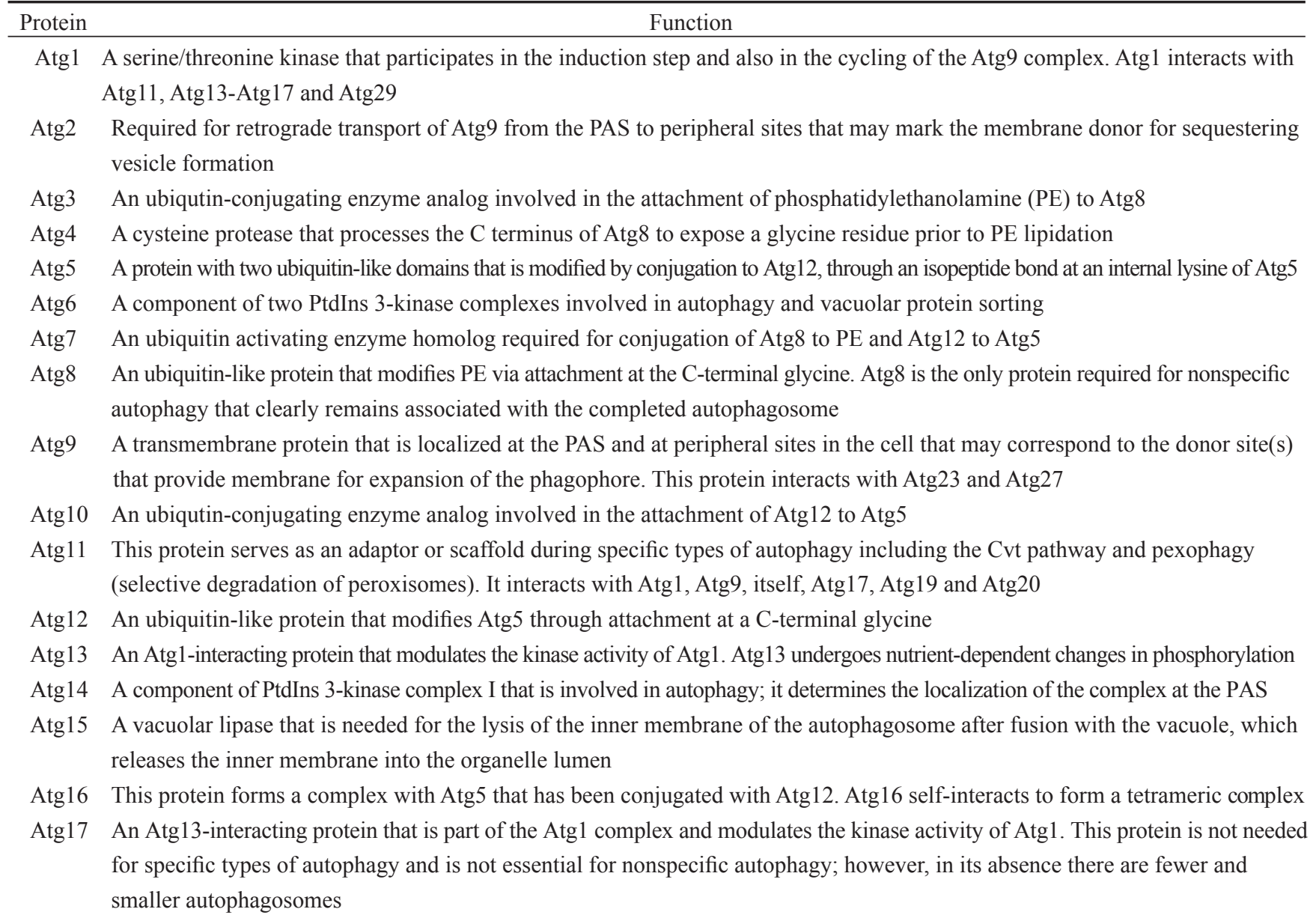

Atg18 Interacts with Atg9 and required for retrograde transport of Atg9 from the PAS to peripheral sites. This protein binds PtdIns(3) phosphate and its localization is dependent on the PtdIns 3-kinase complex

Atg19 A receptor for the substrates of the Cvt pathway that interacts with Atg11; this protein is not needed for nonspecific autophagy

Atg20 A PX domain-containing protein that binds PtdIns(3)phosphate and is required for the Cvt pathway, but not nonspecific autophagy. This protein binds Atg11, Atg17 and Atg24, and is therefore part of a putative Atg1 complex

Atg21 A homolog of Atg18 that also binds PtdIns(3)phosphate; this protein is only essential for the Cvt pathway

Atg22 A vacuolar permease that functions in the final step of autophagy, the release of macromolecules (amino acids) generated by vacuolar degradation back into the cytosol

Atg23 A cytosolic protein that displays a localization pattern and cycles similar to, and interacts with, Atg9. This protein is needed for the Cvt pathway and for efficient nonspecific autophagy; an atg23 $\Delta$ mutant forms a reduced number of autophagosomes

Atg24 A PX domain-containing protein that binds PtdIns(3)phosphate and is required for the Cvt pathway, but not nonspecific autophagy. This protein binds Atg17 and Atg20, and is therefore part of a putative Atg1 complex

Atg25 This protein is not found in S. cerevisiae, but it is required for specific peroxisome degradation in Hansenula polymorpha

Atg26 A GRAM domain-containing protein that binds PtdIns(4)phosphate; this protein is required for specific peroxisome degradation in Pichia pastoris, but not in S. cerevisiae

Atg27 A transmembrane protein that displays a localization pattern and cycles similar to, and interacts with, Atg9. This protein is needed for the Cvt pathway and for efficient nonspecific autophagy; an atg27 mutant forms a reduced number of autophagosomes

Atg28 A coiled-coil domain protein required for specific peroxisome degradation in $P$. pastoris

Atg29 A component of the Atg1 complex that interacts directly with Atg1

Atg30 A protein required for specific peroxisome degradation in P. pastoris

Atg31 A component of the Atg1 complex that interacts directly with Atg17 

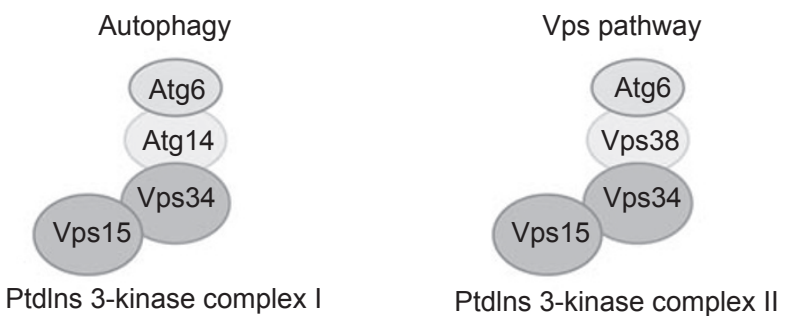

Figure 2 Two PtdIns 3-kinase complexes function in autophagy and the Vps pathway in yeast. Vps34 is the only PtdIns 3-kinase in yeast, but this enzyme is present in two distinct complexes. Both complexes contain Vps34, Vps15, which is thought to be a regulatory factor, and Atg6/Vps30. Complex I and II additionally include Atg14 and Vps38, respectively, which act as connectors between Atg6/Vps30 and the Vps34-Vps15 core. Complex I functions in autophagy, whereas complex II is required for vacuolar protein sorting. Drawing modified from Kihara et al. [32].

promotes autophagy and inhibits in vitro clonigenicity and tumorigenesis in nude mice [41]. Beclin 1 is a haploinsufficient tumor suppressor gene in mice [16, 17], sharing 98\% identity with human beclin 1. Homozygous beclin $1^{-/}$mice die in early embryogenesis, and heterozygous beclin $1^{+/-}$mice have reduced autophagy levels and increased incidence of spontaneous tumors, which, together with the in vitro data, establishes a clear role for autophagy, and Beclin 1, in tumor suppression.

A function for Beclin 1 in tumor suppression is further supported by the identification of additional Beclin 1 -interacting proteins. The UV irradiation resistance-associated gene protein, UVRAG, is a positive regulator of the Beclin 1-PtdIns 3-kinase complex; UVRAG promotes autophagy in a manner that is interdependent with Beclin 1 [42]. UVRAG is monoallelically deleted in various human cancers, similar to beclin 1, suggesting that the UVRAG protein is a tumor suppressor, and expression of UVRAG inhibits the proliferation and tumorigenicity of human colon cancer cells. The activating molecule in Beclin 1-regulated autophagy, Ambra1, is another Beclin 1-interacting protein that positively regulates autophagy and inhibits cell proliferation (see below).

In contrast to beclin $1^{-/}$mice, $\operatorname{atg} 5^{-/}$and $\operatorname{atg} 7^{-/}$mice appear almost normal at birth, but die within 1 day after birth $[43,44]$. Furthermore, heterozygous atg $5^{+/}$mice seem normal during a monitoring period of up to 16 months of age [44], and neither tumorigenesis nor enhanced cell proliferation is detected in Atg7-deficient liver [43]. These studies suggest that Beclin 1 plays a more important role in embryogenesis and tumor suppression than Atg5 and Atg7, and/or the autophagy-independent aspects of Beclin 1 may contribute to these functions. However, it is worth noting that a truncated calpain-cleaved form of Atg5 induces apoptosis and sensitizes tumor cells to anticancer drug treatment [45].

Human Beclin 1 shares $24.4 \%$ identity with Atg6/Vps30 in yeast. It complements the autophagy function, but not the Vps function of atg6/vps30 mutant yeast [41], and a Vps function for Beclin 1 in mammalian cells has not been established. Maturation of the lysosomal enzyme cathepsin $\mathrm{D}$ is normal in MCF7 cells, which have undetectable or very low levels of Beclin 1 [46]. Similarly, knockdown of Beclin 1 in U-251 glioma cells does not affect cathepsin D processing (i.e., lysosomal delivery) and endocytic trafficking [47], suggesting that some other protein may fulfill the $\mathrm{Vps}$ role of Atg6/Vps30 in mammalian cells. However, it is still possible that very low levels of Beclin 1 are sufficient for its Vps function, and Beclin 1 might have an unidentified vesicular trafficking function. Endogenous Beclin 1 in HeLa cells localizes predominantly to the trans-Golgi network [48], but endogenous Beclin 1 also colocalizes with mitochondria and endoplasmic reticulum (ER) in HT-29 cells [49] in agreement with data from overexpressed protein in COS7 and MCF7 cells [50]. It is possible that Beclin 1 has a slightly different localization in different cell lines, and it has a subpopulation that localizes to mitochondria and ER. In fact, recent studies suggest that ER-localized Beclin 1 plays a role in regulating autophagy [51], although other populations of Beclin 1 may also regulate this pathway.

Sequence and structural studies indicate that Beclin 1 has a BH3-only domain, a central coiled-coil domain (CCD), and an evolutionarily conserved domain (ECD) (Figure 3 ). The ECD of Beclin 1 is essential for Vps34 binding, autophagy and the tumor suppressor function [46]. BH3 proteins are part of the Bcl-2 family; they are pro-apoptotic damage sensors that play an important role in protecting

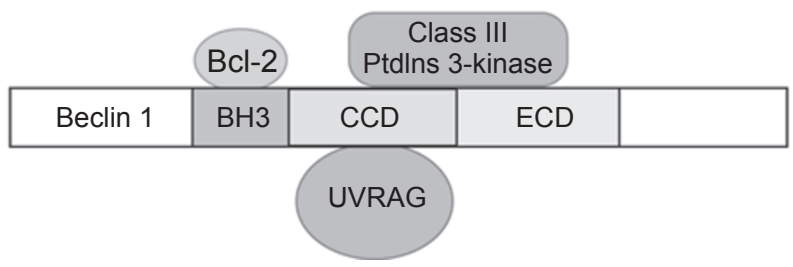

Figure 3 Schematic representations of domains of human Beclin 1 and the Bcl-2-Beclin 1-PtdIns 3-kinase-UVRAG multiprotein complex. Beclin 1 has a BH3 domain (amino acids 114-123), a central coiled-coil domain (CCD, amino acids 144-269) and an evolutionarily conserved domain (ECD, amino acids 244-337). Bcl-2 interacts with the BH3 domain of Beclin 1, UVRAG with the CCD, and the class III PtdIns 3-kinase with the ECD and CCD. Beclin 1 functions as a platform or scaffold for the formation of the complex. Drawing modified from Liang et al. [42]. 
against cancer [52]. The BH3-only domain of Beclin 1 can interact with Bcl-2 and $\mathrm{Bcl}-\mathrm{X}_{\mathrm{L}}[19,20]$, although one study shows that in U-251 cells endogenous Beclin 1 does not interact with either protein [47]. Both cellular and viral Bcl-2 (vBcl-2), or more specifically ER-targeted Bcl-2, inhibit Beclin 1-dependent autophagy by interfering with the Beclin 1-PtdIns 3-kinase interaction and the Beclin 1-associated PtdIns 3-kinase activity [19, 49]. The interaction between Bcl-2 and Beclin 1 is greatly reduced upon starvation, which suggests that the dissociation of $\mathrm{Bcl}-2$ from Beclin 1 is important for activating autophagy.

UVRAG interacts with the CCD of Beclin 1 through its own central CCD [42]. Unlike the interaction between Bcl-2 and Beclin 1, the UVRAG-Beclin 1 interaction is not affected by starvation. In the multiprotein complex vBcl-2-Beclin 1-PtdIns 3-kinase-UVRAG, Beclin 1 acts as a platform mediating interaction between $\mathrm{vBcl}-2$ and UVRAG, between vBcl-2 and PtdIns 3-kinase, and between UVRAG and PtdIns 3-kinase (Figure 3). UVRAG expression increases the Beclin 1-PtdIns 3-kinase interaction and class III PtdIns 3-kinase activity, which promotes autophagy and suppresses growth and tumorigenesis of HCT116 cells.

Ambra1, a novel activating molecule in Beclin 1-regulated autophagy that is unique to vertebrates, positively regulates autophagy and plays a role in neural tube development and embryogenesis [53]. Ambral interacts with Beclin 1 and is in a complex with Beclin 1 and Vps34. Ambra1 overexpression upregulates autophagy and decreases the cell proliferation rate, whereas its downregulation by siRNA decreases rapamycin- and starvation-induced autophagy, impairs interaction between Beclin 1 and Vps34, and increases cell proliferation. Ambra1 null mouse embryos have impaired autophagy and exhibit excessive cell proliferation followed by increased apoptosis. These data support a role of autophagy in neuronal development, in regulating cell proliferation and in cell death.

Beclin 1 levels appear to be one of the critical factors that affect the induction of autophagy. As indicated above, beclin 1 is haploinsufficient, and various cancer cells show decreased levels of Beclin 1 [39]. The anti-cancer drug tamoxifen may work in part by increasing expression of this protein [54]. Other experiments also support the importance of Beclin 1 levels in autophagy regulation. For example, ceramide is thought to play a role in apoptosis $[55,56]$. Recent studies show that ceramide can also induce autophagy, and that it may be involved in the tamoxifendependent increase in Beclin 1 levels [57]. Altered levels of Beclin 1 are also seen in situations other than cancer that involve autophagy; high levels of Beclin 1 are associated with neurons at the site of traumatic brain injury [58], whereas inhibition of Beclin 1 expression protects against cell death due to ischemia/reperfusion [59].

\section{Beclin 1-mediated autophagy and other pathologies}

Autophagy also plays a role in diseases other than cancer. For example, expression of Beclin 1 is important for reducing protein aggregates [60], which supports a general protective role of autophagy and Beclin 1 in neurodegeneration [9]. On the other hand, autophagic degradation may contribute to tissue damage in sporadic inclusion body myositis [61, 62]. Similarly, as discussed above, Beclin 1 functions in autophagy as part of a class III PtdIns 3-kinase/ Vps34 complex [48]. Amino acid withdrawal stimulates class III PtdIns 3-kinase activity associated with Beclin 1 [63], which further induces autophagy in $\mathrm{C}_{2} \mathrm{C}_{12}$ myotubes. Thus, Beclin 1-dependent autophagy may contribute to the degradation that is associated with certain myopathies.

The dual nature of autophagy in cytoprotection and cell death is also seen in ischemia/reperfusion. Autophagy is induced during ischemia in an AMPK-dependent manner, whereas during reperfusion it is accompanied by upregulation of Beclin 1 [64]. Autophagy plays a protective role during ischemia, whereas cardiac injury associated with reperfusion is attenuated in beclin $1^{+/}$mice. Thus, Beclin 1-mediated autophagy during reperfusion can be detrimental in heart cells.

Although some data have implicated autophagy as playing a role in neuronal cell death $[65,66]$, most evidence indicates that autophagy generally serves a cytoprotective role in neurons. For example, otherwise healthy mice specifically lacking neuronal Atg5 or Atg7 develop symptoms of neurodegeneration $[67,68]$. Similarly, autophagy plays a role in preventing Huntington's disease, spinocerebellar ataxia and some forms of familial Parkinson's disease [67, 68]. Even in C. elegans, Beclin 1-dependent autophagy plays a role in preventing neurodegeneration associated with the expression of polyQ-containing fragments of huntingtin [69].

Upregulation of autophagy mediated by increased expression of Beclin 1 is observed in a mouse model for Niemann-Pick C (NPC) disease [70], which is characterized by lipid trafficking defects and neurodegeneration due to defects in the NPC1 gene. Similar induction of autophagy and Beclin 1 expression is observed in wild-type fibroblasts treated with U18666A (a compound that causes accumulation of unesterified cholesterol) and in primary fibroblast cells from patients with NPC2 deficiency and Sandhoff disease, two other sphingolipid storage diseases. The implications of these findings are not clear, but they suggest that Beclin 1-dependent autophagy may be induced as a cytoprotective response in some lipid-trafficking diseases. It is also possible, however, that autophagic induction is 
an indirect effect of sphingolipid accumulation because sphingolipids regulate autophagy [57].

Beclin 1-mediated autophagy also protects against viral infection. Overexpression of Beclin 1 protects mice against lethal Sindbis virus encephalitis [18]. Autophagy also is involved in the clearance of the herpes simplex virus type 1 (HSV-1) from infected host cells [71]. The HSV-1 neurovirulence protein ICP34.5 inhibits autophagy through its binding to Beclin 1 [72], suggesting that endogenous amounts of Beclin 1 are important in protecting against viral invasion. HSV-1 mutants encoding ICP34.5 proteins that are unable to bind Beclin 1 are neuroattenuated in mice, whereas neurovirulence is restored in $p k r^{-/}$mice, which are defective in double-stranded RNA-activated protein kinase R, PKR, signaling that normally activates autophagy upon viral infection. Increased viral replication is also observed in atg6-silenced plants [73].

\section{Atg6 and the hypersensitive response in plants}

Autophagy plays a role in innate and adaptive immunity. For example, autophagy defends cells against invasive bacteria and viruses [71, 74-76], and is involved in major histocompatibility complex II antigen presentation [77, 78]. Similarly, autophagy plays a role in innate immunity in plants. The plant orthologue of yeast Atg6 was first identified in a screen for genes that affect tobacco mosaic virus-induced hypersensitive response PCD (HR PCD) in Nicotiana tabacum $(\mathrm{Nb})$ using a virus-induced genesilencing method [73]. Plants induce the HR at sites of pathogen infection; PCD of infected cells limits the spread of the pathogen. In normal plants, HR PCD is restricted to the infection site and does not spread to neighboring cells, whereas cell death extends to uninfected tissue and distal uninfected leaves in the NbATG6-deficient plant indicating that autophagy is involved in negatively regulating PCD. In addition to NbATG6, orthologues of VPS34, $A T G 3$ and $A T G 7$ are also required to restrict HR PCD [73]. Furthermore, in $N b A T G$-silenced plants, there is increased accumulation of tobacco mosaic virus at infection sites, suggesting a role for $A T G 6$ and other $A T G$ genes in controlling virus replication.

NbATG6 shares 69\% identity with Arabidopsis ATG6 (AtATGO), 36\% identity with human beclin 1 and 24\% identity with yeast $A T G 6$. No apparent difference is observed between the phenotypes of NbATG6-silenced and other $N b A T G$-silenced plants, and no obvious developmental defect is noted [73]. In contrast, mutants in Arabidopsis thaliana ATG6 display defects in pollen germination [14, 15]. Disruption of AtATG6 by T-DNA insertion results in male sterility, whereas all previously characterized Atatg mutants are fertile. These results indicate that autophagy may not contribute to the pollen germination defect in the AtATG6-deficient plants. In contrast to mammalian beclin 1, expression of AtATG6 restores both autophagy and Vps in atg6/vps30 mutant yeast [14]; vesicular trafficking is involved in pollen tube growth, and hence it is proposed that the Vps function of AtAtg6 is responsible for pollen germination. With regard to NbAtg6, it is possible that a residual level of activity resulting from the gene-silencing system used in that analysis may be sufficient for normal development to occur [73]. It will be interesting to know whether NbATG6 can complement the Vps defect in atg6/vps 30 mutant yeast, and to determine whether NbATG6-deficient, rather than NbATG6-silenced, plants are also defective in pollen germination. Nonetheless, all the data point to a general role of autophagy genes, and autophagy, in regulating HR PCD during the plant innate immune response.

\section{BEC-1 and life span extension in C. elegans}

One of the primary factors that correlate with longevity is caloric restriction [79]. The general idea behind this observation is that the housekeeping function of autophagy that allows the removal of damaged proteins and organelles is critical in maintaining cell and organism viability. Studies with rats demonstrate that autophagy declines with age and that stimulation of autophagy in older organisms has beneficial effects [80]. Nonetheless, there are obviously complicating factors in carrying out these types of analyses and drawing definitive conclusions from studies in mammals; however, work from C. elegans supports the view that autophagy plays a role in life span extension. The $C$. elegans $B E C-1$ gene shares $28 \%$ homology with yeast $A T G 6 / V P S 30$ and $31 \%$ homology with human beclin 1 [81]. Expression of $B E C-1$ complements the autophagy, but not the Vps defect of the yeast atg6/vps30 mutant. Knockdown of autophagy genes including $B E C-1$ inhibits autophagy and blocks normal dauer formation (a stage of developmental arrest under unfavorable conditions) in $C$. elegans [81], and bec-1 mutants display a range of developmental defects [82]. BEC-1 is also required for life span extension in this organism $[81,83]$. BEC-1 interacts with LET-512, the orthologue of the PtdIns 3-kinase Vps34, and is needed for the function of the enzyme; bec-1 mutants are defective in membrane trafficking [82], suggesting that the C. elegans protein may function in two different complexes, one involved in protein sorting and the other in autophagy, similar to yeast Atg6.

\section{Beclin 1 in autophagic cell death and apoptosis}

In addition to its role in autophagy, development, tumor 
suppression and the clearance of aggregate-prone proteins, Beclin 1 also serves as an important regulator or player in PCD. For example, Beclin 1 is required for autophagic, type II, PCD [84]. As indicated above, mammalian Beclin 1 binds Bcl-2 [18], and similarly, the C. elegans Bcl-2 orthologue, CED-9, also binds Beclin 1 [82]. Bcl-2 inhibits Beclin 1-dependent autophagy, and thus autophagic cell death upon starvation $[19,49]$. However, overexpression of Bcl-2 or Bcl- $\mathrm{X}_{\mathrm{L}}$ in apoptosis-defective bax $^{-/}$bak $^{-/-}$mouse embryonic fibroblast cells treated with etoposide, an apoptosis-inducing agent, stimulates Beclin 1-dependent autophagic cell death [85]. These findings suggest that Bcl2 has a different effect on autophagy and autophagic cell death depending on the cell type and stimulus, and are in agreement with the general observation that autophagy can act in both cytoprotective and cell death functions. In addition, the level of $\mathrm{Bcl}-2$ proteins may need to be precisely regulated to ensure proper control over autophagy and apoptosis; however, in physiological conditions it seems that Bcl-2 has an inhibitory effect on autophagy. Additional experiments point to the connection between autophagy and apoptosis. For example, inhibition of autophagy with small interfering RNA to reduce levels of Beclin 1, or other autophagy proteins, leads to apoptotic cell death, and the reduction in autophagy sensitizes cells to apoptotic stimuli, suggesting some type of crosstalk between these pathways $[86,87]$. Depletion of Beclin 1 through RNA interference in C. elegans also results in elevated apoptosis, and similar effects are seen in homozygous bec- $1^{-/}$animals [82]. In general, autophagy probably acts initially as a cytoprotective mechanism, and when autophagy is suppressed, cells are more prone to apoptosis. On the other hand, if cell death occurs when apoptosis is blocked, it is likely to involve an autophagic mechanism.

Beclin 1 is not essential for apoptosis, although it has recently been found to have a $\mathrm{BH} 3$ domain, which classifies it as a BH3-only protein. The other known members of the BH3-only family (e.g., Bid and Bad) are considered to function in a pro-apoptotic manner, upstream of anti-apoptotic Bcl-2-like proteins [20]. These BH3-only proteins activate Bax-like apoptotic factors (including Bax and Bak), which cause changes in mitochondrial permeability that lead to apoptosis. Along these lines, a synthetic peptide containing the $\mathrm{BH} 3$ domain of Beclin 1 induces apoptosis [19]; however, it is important to note that the intact protein, even upon overexpression, does not promote apoptosis. Moreover, in embryonic stem cells derived from beclin $1^{-/-}$mice, apoptotic cell death induced by UV light or serum deprivation is not compromised [17]. One implication of these findings is that the $\mathrm{BH} 3$ domain of Beclin 1 in the native protein is not presented in a similar manner as other BH3-only proteins. Therefore, Beclin 1 could be a unique member of the $\mathrm{BH} 3$-only proteins, the function of which could be regulated by other BH3-only proteins. For example, BH3-only proteins such as Bad may induce autophagy by competitively disrupting the interaction between Beclin 1 and $\mathrm{Bcl}-2 / \mathrm{Bcl}-\mathrm{X}_{\mathrm{L}}$, in addition to their pro-apoptotic roles.

As autophagy and apoptosis are interconnected, and the relationship between them may vary depending on the particular context, it is possible that Beclin 1 might have a regulatory role in apoptosis and other related cellular events. For instance, using an in vitro mouse model of embryogenesis, a recent study shows that beclin 1 is not required for apoptotic cell death, but for the generation of signals that allow the phagocytic clearance of apoptotic corpses [88]. As atg 5 $5^{-/}$embryoid bodies (EB) have the same phenotypes as beclin $1^{-/ \mathrm{EB}}$, the role for clearance of cell corpses is not specific to Beclin 1. It is unexpected that both $\operatorname{atg} 5^{--}$and beclin $1^{-/}$EBs fail to cavitate, given that only beclin $1^{-/}$mice are embryonic lethal $[16,17,44]$; thus, this in vitro study does not fully replicate the in vivo situation.

\section{Conclusions and future directions}

Atg6/Beclin 1 has roles in many cellular processes, some of which involve autophagy-independent functions. Future studies need to separate Atg6/Beclin 1-specific functions from its general participation in autophagy. The search for Atg14 and Vps38 homologs in mammals will help to establish the different roles, if any, of Beclin 1 in autophagy and lysosomal protein sorting. Additionally, the identification of other Atg6/Beclin 1 interaction partners may provide important information concerning any non-autophagic roles. It is possible that different Beclin 1 complexes exist, and exert their function at different cellular locations or under different stimuli. Generation of knock-in mice expressing mutant Beclin 1 that loses interactions with specific partners is another approach that may help to dissect the function of Beclin 1. Research on Atg6/Beclin 1 will continue to provide insights into the mechanisms of autophagy, the crosstalk between autophagy and apoptosis, and the role of autophagy and Atg6/Beclin 1 in many important cellular processes.

\section{Acknowledgment}

This work was supported by the USA National Institutes of Health Public Health Service Grant GM53396.

\section{References}

1 Klionsky DJ, Cuervo AM, Dunn WA Jr, et al. How shall I eat 
thee? Autophagy 2007; 3:413-416.

2 Klionsky DJ. The molecular machinery of autophagy: unanswered questions. J Cell Sci 2005; 118:7-18.

3 Levine B, Klionsky DJ. Development by self-digestion: molecular mechanisms and biological functions of autophagy. Dev Cell 2004; 6:463-477.

4 Li C, Capan E, Zhao Y, et al. Autophagy is induced in $\mathrm{CD}^{+} \mathrm{T}$ cells and important for the growth factor-withdrawal cell death. J Immunol 2006; 177:5163-5168.

5 Levine B. Eating oneself and uninvited guests: autophagy-related pathways in cellular defense. Cell 2005; 120:159-162.

6 Mathew R, Kongara S, Beaudoin B, et al. Autophagy suppresses tumor progression by limiting chromosomal instability. Genes Dev 2007; 21:1367-1381.

7 Zhang Y, Qi H, Taylor R, et al. The role of autophagy in mitochondria maintenance: characterization of mitochondrial functions in autophagy-deficient S. cerevisiae strains. Autophagy 2007; 3:337-346.

8 Gozuacik D, Kimchi A. Autophagy as a cell death and tumor suppressor mechanism. Oncogene 2004; 23:2891-2906.

9 Rubinsztein DC, Difiglia M, Heintz N, et al. Autophagy and its possible roles in nervous system diseases, damage and repair. Autophagy 2005; 1:11-22.

10 Rubinsztein DC, Gestwicki JE, Murphy LO, Klionsky DJ. Potential therapeutic applications of autophagy. Nature Rev Drug Discov 2007; 6:304-312.

11 Shintani T, Klionsky DJ. Autophagy in health and disease: a double-edged sword. Science 2004; 306:990-995.

12 Klionsky DJ, Cregg JM, Dunn WA Jr, et al. A unified nomenclature for yeast autophagy-related genes. Dev Cell 2003; 5:539545 .

13 Kametaka S, Okano T, Ohsumi M, Ohsumi Y. Apg14p and Apg6/ $\mathrm{Vps} 30 \mathrm{p}$ form a protein complex essential for autophagy in the yeast, Saccharomyces cerevisiae. J Biol Chem 1998; 273:2228422291.

14 Fujiki Y, Yoshimoto K, Ohsumi Y. An Arabidopsis homolog of yeast $A T G 6 / V P S 30$ is essential for pollen germination. Plant Physiol 2007; 143:1132-1139.

15 Qin G, Ma Z, Zhang L, et al. Arabidopsis AtBECLIN 1/AtAtg6/ AtVps30 is essential for pollen germination and plant development. Cell Res 2007; 17:249-263.

$16 \mathrm{Qu} \mathrm{X,} \mathrm{Yu} \mathrm{J,} \mathrm{Bhagat} \mathrm{G,} \mathrm{et} \mathrm{al.} \mathrm{Promotion} \mathrm{of} \mathrm{tumorigenesis} \mathrm{by}$ heterozygous disruption of the beclin 1 autophagy gene. J Clin Invest 2003; 112:1809-1820.

17 Yue Z, Jin S, Yang C, Levine AJ, Heintz N. Beclin 1, an autophagy gene essential for early embryonic development, is a haploinsufficient tumor suppressor. Proc Natl Acad Sci USA 2003; 100:15077-15082.

18 Liang XH, Kleeman LK, Jiang HH, et al. Protection against fatal Sindbis virus encephalitis by beclin, a novel Bcl-2-interacting protein. J Virol 1998; 72:8586-8596.

19 Maiuri MC, Le Toumelin G, Criollo A, et al. Functional and physical interaction between $\mathrm{Bcl}-\mathrm{X}_{\mathrm{L}}$ and a $\mathrm{BH} 3$-like domain in Beclin-1. EMBO J 2007; 26:2527-2539.

20 Oberstein A, Jeffrey PD, Shi Y. Crystal structure of the Bcl- $\mathrm{X}_{\mathrm{L}}$ Beclin 1 peptide complex: Beclin 1 is a novel $\mathrm{BH} 3$-only protein. J Biol Chem 2007; 282:13123-13132.

21 Huang W-P, Klionsky DJ. Autophagy in yeast: a review of the molecular machinery. Cell Struct Funct 2002; 27:409-420.
22 Klionsky DJ, Cuervo AM, Seglen PO. Methods for monitoring autophagy from yeast to human. Autophagy 2007; 3:181-206.

23 Wang C-W, Klionsky DJ. The molecular mechanism of autophagy. Mol Med 2003; 9:65-76.

24 Yorimitsu T, Klionsky DJ. Autophagy: molecular machinery for self-eating. Cell Death Differ 2005; 12:1542-1552.

25 Dunn WA Jr, Cregg JM, Kiel JAKW, et al. Pexophagy: the selective autophagy of peroxisomes. Autophagy 2005; 1:75-83.

26 Nair U, Klionsky DJ. Molecular mechanisms and regulation of specific and nonspecific autophagy pathways in yeast. J Biol Chem 2005; 280:41785-41788.

27 Harding TM, Hefner-Gravink A, Thumm M, Klionsky DJ. Genetic and phenotypic overlap between autophagy and the cytoplasm to vacuole protein targeting pathway. J Biol Chem 1996; 271:17621-17624.

28 Scott SV, Hefner-Gravink A, Morano KA, et al. Cytoplasm-tovacuole targeting and autophagy employ the same machinery to deliver proteins to the yeast vacuole. Proc Natl Acad Sci USA 1996; 93:12304-12308.

29 Dove SK, Piper RC, McEwen RK, et al. Svp1p defines a family of phosphatidylinositol 3,5-bisphosphate effectors. EMBO J 2004; 23:1922-1933.

30 Horazdovsky BF, DeWald DB, Emr SD. Protein transport to the yeast vacuole. Curr Opin Cell Biol 1995; 7:544-551.

31 Petiot A, Ogier-Denis E, Blommaart EF, Meijer AJ, Codogno P. Distinct classes of phosphatidylinositol 3'-kinases are involved in signaling pathways that control macroautophagy in HT-29 cells. J Biol Chem 2000; 275:992-998.

32 Kihara A, Noda T, Ishihara N, Ohsumi Y. Two distinct Vps34 phosphatidylinositol 3-kinase complexes function in autophagy and carboxypeptidase Y sorting in Saccharomyces cerevisiae. J Cell Biol 2001; 152:519-530.

33 Obara K, Sekito T, Ohsumi Y. Assortment of phosphatidylinositol 3-kinase complexes - Atg14p directs association of complex I to the pre-autophagosomal structure in Saccharomyces cerevisiae. Mol Biol Cell 2006; 17:1527-1539.

34 Kim J, Huang W-P, Stromhaug PE, Klionsky DJ. Convergence of multiple autophagy and cytoplasm to vacuole targeting components to a perivacuolar membrane compartment prior to de novo vesicle formation. J Biol Chem 2002; 277:763-773.

35 Suzuki K, Kirisako T, Kamada Y, et al. The pre-autophagosomal structure organized by concerted functions of $A P G$ genes is essential for autophagosome formation. EMBO J 2001; 20:59715981.

36 Suzuki K, Kubota Y, Sekito T, Ohsumi Y. Hierarchy of Atg proteins in pre-autophagosomal structure organization. Genes Cells 2007; 12:209-218.

37 Walensky LD. BCL-2 in the crosshairs: tipping the balance of life and death. Cell Death Differ 2006; 13:1339-1350.

38 Aita VM, Liang XH, Murty VVVS, et al. Cloning and genomic organization of beclin 1, a candidate tumor suppressor gene on chromosome 17q21. Genomics 1999; 59:59-65.

39 Miracco C, Cosci E, Oliveri G, et al. Protein and mRNA expression of autophagy gene Beclin 1 in human brain tumours. Int $\mathrm{J}$ Oncol 2007; 30:429-436.

40 Wang ZH, Xu L, Duan ZL, et al. Beclin 1-mediated macroautophagy involves regulation of caspase- 9 expression in cervical cancer HeLa cells. Gynecol Oncol 2007.

41 Liang XH, Jackson S, Seaman M, et al. Induction of autoph- 
agy and inhibition of tumorigenesis by beclin 1. Nature 1999; 402:672-676.

42 Liang C, Feng P, Ku B, et al. Autophagic and tumour suppressor activity of a novel Beclin1-binding protein UVRAG. Nat Cell Biol 2006; 8:688-699.

43 Komatsu M, Waguri S, Ueno T, et al. Impairment of starvationinduced and constitutive autophagy in Atg7-deficient mice. J Cell Biol 2005; 169:425-434.

44 Kuma A, Hatano M, Matsui M, et al. The role of autophagy during the early neonatal starvation period. Nature 2004; 432:10321036.

45 Yousefi S, Perozzo R, Schmid I, et al. Calpain-mediated cleavage of Atg5 switches autophagy to apoptosis. Nat Cell Biol 2006; 8:1124-1132.

46 Furuya N, Yu J, Byfield M, Pattingre S, Levine B. The evolutionarily conserved domain of Beclin 1 is required for $\mathrm{Vps} 34$ binding, autophagy and tumor suppressor function. Autophagy 2005; 1:46-52.

47 Zeng X, Overmeyer JH, Maltese WA. Functional specificity of the mammalian Beclin-Vps34 PI 3-kinase complex in macroautophagy versus endocytosis and lysosomal enzyme trafficking. J Cell Sci 2006; 119:259-270.

48 Kihara A, Kabeya Y, Ohsumi Y, Yoshimori T. Beclin-phosphatidylinositol 3-kinase complex functions at the trans-Golgi network. EMBO Rep 2001; 2:330-335.

49 Pattingre S, Tassa A, Qu X, et al. Bcl-2 antiapoptotic proteins inhibit Beclin 1-dependent autophagy. Cell 2005; 122:927-939.

50 Liang XH, Yu J, Brown K, Levine B. Beclin 1 contains a leucinerich nuclear export signal that is required for its autophagy and tumor suppressor function. Cancer Res 2001; 61:3443-3449.

51 Maiuri MC, Criollo A, Tasdemir E, et al. BH3-only proteins and $\mathrm{BH} 3$ mimetics induce autophagy by competitively disrupting the interaction between Beclin 1 and $\mathrm{Bcl}-2 / \mathrm{Bcl}-\mathrm{X}_{\mathrm{L}}$. Autophagy 2007; 3:374-376.

52 Karst AM, Li G. BH3-only proteins in tumorigenesis and malignant melanoma. Cell Mol Life Sci 2007; 64:318-330.

53 Maria Fimia G, Stoykova A, Romagnoli A, et al. Ambra1 regulates autophagy and development of the nervous system. Nature 2007; 447:1121-1125.

54 Furuya N, Liang XH, Levine B. Autophagy and cancer. In: Klionsky DJ. ed. Autophagy. Georgetown, TX: Landes Bioscience, 2004.

55 Levade T, Malagarie-Cazenave S, Gouaze V, et al. Ceramide in apoptosis: a revisited role. Neurochem Res 2002; 27:601-607.

56 Pettus BJ, Chalfant CE, Hannun YA. Ceramide in apoptosis: an overview and current perspectives. Biochim Biophys Acta 2002; 1585:114-125.

57 Scarlatti F, Bauvy C, Ventruti A, et al. Ceramide-mediated macroautophagy involves inhibition of protein kinase B and upregulation of beclin 1. J Biol Chem 2004; 279:18384-18391.

58 Diskin T, Tal-Or P, Erlich S, et al. Closed head injury induces upregulation of Beclin 1 at the cortical site of injury. J Neurotrauma 2005; 22:750-762.

59 Valentim L, Laurence KM, Townsend PA, et al. Urocortin inhibits Beclin1-mediated autophagic cell death in cardiac myocytes exposed to ischaemia/reperfusion injury. J Mol Cell Cardiol 2006; 40:846-852.

60 Shibata M, Lu T, Furuya T, et al. Regulation of intracellular accumulation of mutant huntingtin by Beclin 1. J Biol Chem 2006;
281:14474-14485.

61 Lunemann JD, Schmidt J, Dalakas MC, Munz C. Macroautophagy as a pathomechanism in sporadic inclusion body myositis. Autophagy 2007; 3:384-386.

62 Lunemann JD, Schmidt J, Schmid D, et al. $\beta$-Amyloid is a substrate of autophagy in sporadic inclusion body myositis. Ann Neurol 2007; 61:476-483.

63 Tassa A, Roux MP, Attaix D, Bechet DM. Class III phosphoinositide 3-kinase-Beclin1 complex mediates the amino acid-dependent regulation of autophagy in $\mathrm{C} 2 \mathrm{C} 12$ myotubes. Biochem $\mathrm{J}$ 2003; 376:577-586.

64 Matsui Y, Takagi H, Qu X, et al. Distinct roles of autophagy in the heart during ischemia and reperfusion: roles of AMP-activated protein kinase and Beclin 1 in mediating autophagy. Circ Res 2007; 100:914-922.

65 Yue Z, Horton A, Bravin M, et al. A novel protein complex linking the $\delta 2$ glutamate receptor and autophagy: implications for neurodegeneration in lurcher mice. Neuron 2002; 35:921-933.

66 Toth ML, Simon P, Kovacs AL, Vellai T. Influence of autophagy genes on ion-channel-dependent neuronal degeneration in Caenorhabditis elegans. J Cell Sci 2007; 120:1134-1141.

67 Hara T, Nakamura K, Matsui M, et al. Suppression of basal autophagy in neural cells causes neurodegenerative disease in mice. Nature 2006; 441:885-889.

68 Komatsu M, Waguri S, Chiba T, et al. Loss of autophagy in the central nervous system causes neurodegeneration in mice. Nature 2006; 441:880-884.

69 Jia K, Hart AC, Levine B. Autophagy genes protect against disease caused by polyglutamine expansion proteins in $\mathrm{Cae}$ norhabditis elegans. Autophagy 2007; 3:21-25.

70 Pacheco CD, Kunkel R, Lieberman AP. Autophagy in NiemannPick C disease is dependent upon Beclin-1 and responsive to lipid trafficking defects. Hum Mol Genet 2007; 16:1495-1503.

71 Talloczy Z, Virgin HW IV, Levine B. PKR-dependent autophagic degradation of herpes simplex virus type 1. Autophagy 2006; 2:24-29.

72 Orvedahl A, Alexander D, Talloczy Z, et al. HSV-1 ICP34.5 confers neurovirulence by targeting the Beclin 1 autophagy protein. Cell Host Microbe 2007; 1:23-25.

73 Liu Y, Schiff M, Czymmek K, et al. Autophagy regulates programmed cell death during the plant innate immune response. Cell 2005; 121:567-577.

74 Birmingham CL, Brumell JH. Autophagy recognizes intracellular Salmonella enterica serovar Typhimurium in damaged vacuoles. Autophagy 2006; 2:156-158.

75 Colombo MI, Gutierrez MG, Romano PS. The two faces of autophagy: Coxiella and Mycobacterium. Autophagy 2006; 2:162-164.

76 Yoshimori T. Autophagy vs. group A Streptococcus. Autophagy 2006; 2:154-155.

77 Paludan C, Schmid D, Landthaler M, et al. Endogenous MHC class II processing of a viral nuclear antigen after autophagy. Science 2005; 307:593-596.

78 Schmid D, Munz C. Immune surveillance of intracellular pathogens via autophagy. Cell Death Differ 2005; 12:1519-1527.

79 Bergamini E. Autophagy: a cell repair mechanism that retards ageing and age-associated diseases and can be intensified pharmacologically. Mol Aspects Med 2006; 27:403-410.

80 Donati A. The involvement of macroautophagy in aging and 
anti-aging interventions. Mol Aspects Med 2006; 27:455-470.

81 Melendez A, Talloczy Z, Seaman M, et al. Autophagy genes are essential for dauer development and life-span extension in $C$. elegans. Science 2003; 301:1387-1391.

82 Takacs-Vellai K, Vellai T, Puoti A, et al. Inactivation of the autophagy gene bec-1 triggers apoptotic cell death in C. elegans. Curr Biol 2005; 15:1513-1517.

83 Hars ES, Qi H, Ryazanov AG, et al. Autophagy regulates ageing in C. elegans. Autophagy 2007; 3:93-95.

$84 \mathrm{Yu} \mathrm{L}$, Alva A, Su H, et al. Regulation of an ATG7-beclin 1 program of autophagic cell death by caspase-8. Science 2004; 304:1500-1502.
85 Shimizu S, Kanaseki T, Mizushima N, et al. Role of Bcl-2 family proteins in a non-apoptotic programmed cell death dependent on autophagy genes. Nat Cell Biol 2004; 6:1221-1228.

86 Boya P, Gonzalez-Polo RA, Casares N, et al. Inhibition of macroautophagy triggers apoptosis. Mol Cell Biol 2005; 25:10251040.

87 Daniel F, Legrand A, Pessayre D, et al. Partial Beclin 1 silencing aggravates doxorubicin- and Fas-induced apoptosis in HepG2 cells. World J Gastroenterol 2006; 12:2895-2900.

$88 \mathrm{Qu}$ X, Zou Z, Sun Q, et al. Autophagy gene-dependent clearance of apoptotic cells during embryonic development. Cell 2007; 128:931-946. 